\title{
A community based authentication and authorisation mechanism for digital ecosystem
}

by llung Pranata, Geoff Skinner \& Rukshan Athauda

Copyright (C) 2011 IEEE.

This is an author-prepared version of the article, reprinted from the Proceedings of the 5th IEEE International Conference on Digital Ecosystems and Technologies Conference (DEST), 2011, p. 158-163.

http://dx.doi.org/10.1109/DEST.2011.5936618

This material is posted here with permission of the IEEE. Such permission of the IEEE does not in any way imply IEEE endorsement of any of University of Newcastle's products or services. Internal or personal use of this material is permitted. However, permission to reprint/republish this material for advertising or promotional purposes or for creating new collective works for resale or redistribution must be obtained from the IEEE by writing to pubs-permissions@ieee.org. By choosing to view this document, you agree to all provisions of the copyright laws protecting it. 


\section{A Community Based Authentication and Authorisation Mechanism for Digital Ecosystem}

\author{
Ilung Pranata \\ Faculty of Science and IT \\ University of Newcastle \\ Callaghan, Australia \\ Ilung.Pranata@newcastle.edu.au
}

\author{
Geoff Skinner \& Rukshan Athauda \\ Faculty of Science and IT \\ University of Newcastle \\ Callaghan, Australia \\ [Geoff.Skinner, Rukshan.Athauda]@ newcastle.edu.au
}

\begin{abstract}
In this paper, we present a distributed mechanism for elevating resource protection in a Digital Ecosystem environment. The dynamic interaction and collaboration between Digital Ecosystem entities poise several main challenges in protecting the resources and information. First, a strong mechanism is needed to ensure only the authentic entities that are able to access the resources. Second, this mechanism must also maintain the confidentiality and integrity of resources over the untrusted network. Unfortunately, the existing mechanisms which focus on providing a centralized protection facade several issues ranging from single point failure to huge administrative burden. Therefore, we present a distributed mechanism to address these challenges. The inclusivity of community based trust approach in our mechanism further promotes its applicability to the Digital Ecosystem environment, on which it is heavily driven by the interacting entities. Public Key Infrastructure is employed to provide a strong protection during its access workflow.
\end{abstract}

Keywords: Digital Ecosystem; Security Architecture; Authentication; Authorisation; Public Key Infrastructure

\section{INTRODUCTION}

The evolution of Digital Ecosystem (DE) concept in information society has recently gained tremendous interest from various established industry practitioners and academia. $\mathrm{DE}$ is considered as an innovative approach to promote the dynamic environment on which routine interactions between its member entities and continuous evolution occur. The DE environment consists of multiple networked organizations that are constantly collaborating and interacting in a self-organized digital infrastructure [1]. This environment is distinctively characterized by its open, loosely coupled, domain clustered and demand driven in nature [2, 3]. Further, a remarkable characteristic of a DE environment is its evolution mechanism which is analogous to the biological ecosystem [4, 5]. This evolution mechanism fabricates only the most suited components to remain in a DE environment while the less relevant components would slowly disappear into obsolescence [6].

A DE environment is attributed with the occurrences of various interrelated entities or Digital Species (DS). These entities are further categorized into two main roles, which are the resource consumer (client) that requests for the resources or services from other entities, and the resource provider that provides the resources or services. However, the dynamic and open interaction between these entities imposes several challenges in protecting the resources and preventing unauthorized entities from consuming resources. Resource provider facades a great challenge to uphold the confidentiality and availability of information due to the occurrences of various shared resources published in a DE environment. Moreover, the existence of multiple resource consumers, in particular the anonymous or partner entities, to access each individual resource complicates the resource protection. Therefore, a strong security mechanism has been portrayed as a critical element for a successful realization of a DE environment.

Although strong protection mechanism is critically needed, the review of current literature in DE has revealed the nonexistence of single mechanism that provides a comprehensive protection towards multiple resources and entities, particularly in the authentication and authorization process. Several approaches in collaborative environments and distributed systems such as Credential Server (CRES) [7], Grid Security Infrastructure (GSI) MyProxy [8], Community Authorization Service (CAS) [9] and Akenti [10] could be adopted for DE. However, their approaches are predominantly relying on a central authorization server which poses several challenges for DE environment such as single server failure, permissions delegation and central server burden. Therefore, our proposed Distributed Resource Protection Mechanism (DRPM) [11, 12] has attempted to protect the DE resources in a distributed approach while addressing the centralized system challenges.

In this paper, we provide a detailed resource protection mechanism in DRPM, and incorporate a community based trust approach for its authentication and authorization process. This approach would promote the engagement of DE community in protecting DE resources while ensuring a fine-grained resource protection is upheld in a distributed approach. We further detail an approach introduced to protect the resource exchange in the proposed mechanism. The ultimate aim is to protect the resources from any unauthorized use in a Digital Ecosystem. The remainder of this paper is organized into five sections. Section 2 presents a brief overview of DRPM. Section 3 describes our extension to include community based trust into DRPM. Further, it also discusses a detailed implementation of DRPM. Section 4 provides an evaluation to our proposed extension, and finally section 5 summarizes our research and suggests the future work of this research. 


\section{OVERVIEW OF DRPM}

The Distributed Resource Protection Mechanism (DRPM) provides a decentralization management of authorization and authentication process of the interacting entities. Such distributed process allows each resource provider to perform its individual client authentication and authorization without the reliance to a particular authorization server in a DE environment. Moreover, a set of client's authorization permissions are assigned in a form of capability token [13]. This token contains the configurable yet protective permission rules that are granted to the client. The dissemination of capability token further permits the delegation of client authorization permissions.

Client authentication in resource provider end-point is facilitated by the creation of a client profile. The client profile contains critical client information for identification purposes, and it is created during the initial resource provisioning. The implementation of client profile further upholds the confidentiality of resources by ensuring only the authorized clients that are able to access the resources. Additionally, the implementation of client profile also facilitates auditing process in the event resource access tracing is required due to the dispute or counterfeiting of information. A resource registration portal is further employed to generate a client profile. This registration portal captures the required, but voluntarily provided, information about the client. Further, the generated client profile is used for authenticating the clients on multiple resources which are provided by individual resource provider. The client profile is maintained in the resource provider end-point. Such implementation of client profile further reduces the duplication of client data on the resource provider client's repository.

Once a client profile is created, a list of client authorization permissions are assigned into the capability token. The access permissions and policies are expressed in XML [14]. Basic design of capability token contains the client profile identifier, resource provider identifier, resource identifier and list of access permissions. Further, a time-stamp can be implemented in the capability token to determine the validity period of a user in accessing the resources. In the event where the trustworthiness of a new user is equivocal, a short-life capability token can be issued. Once the trustworthiness of the user gradually increases, resource provider can replace the short-life token with longer time-stamp validity. Once it is created, the capability token is disseminated to the requesting client. To ensure the confidentiality and integrity of capability token, Public Key Infrastructure (PKI) [15] is utilized to encrypt the message before it is sent to the requesting client. Further, hashing mechanism is utilized to secure the capability token from any modification on its content.

On every authentication process, the client would be required to provide his certificate and a capability token which contains his client profile identifier for authentication purposes. Client certificate could be obtained from his Certificate Authority (CA). This mechanism ensures two factor client authentication procedures which further enhances the protection of resources at resource provider end-point. The workflow process of a requesting client in DRPM is showed in figure ${ }^{*}$

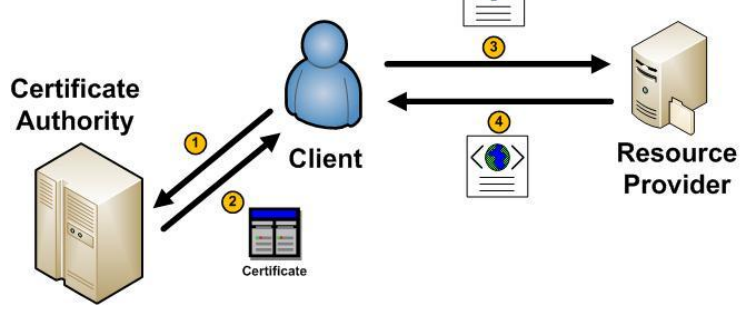

Fig. 1 Authentication workflow for the requesting client

When a client makes a request for a specific resource, client retrieves his certificate from a CA. This CA must be trusted by the resource provider. The implementation of a CA ensures the trustworthiness of both clients and resource providers. Once a client successfully retrieves his certificate, he then binds his certificate and capability token. Public Key Infrastructure is used to encrypt the binding message. This binding message is then sent to the resource provider.

At resource provider end-point, it receives and decrypts the request message. Resource provider authenticates a client by validating a client certificate, and then it authenticates the capability token (by verifying its digital signature. If authentication fails, access is denied and client is redirected to the registration page, otherwise, an authorization process is pursued. In the authorization process, hashing checksum of the capability token is derived to verify the listed permissions have not been modified. This verification is critical to counteract any unauthorized actions that modify the original permissions. Further, a checking on the current capability token time-stamp is conducted to verify whether permissions have changed since the capability token was issued. Once the verification process is completed, client will be granted access based on the permissions retrieved from his capability token.

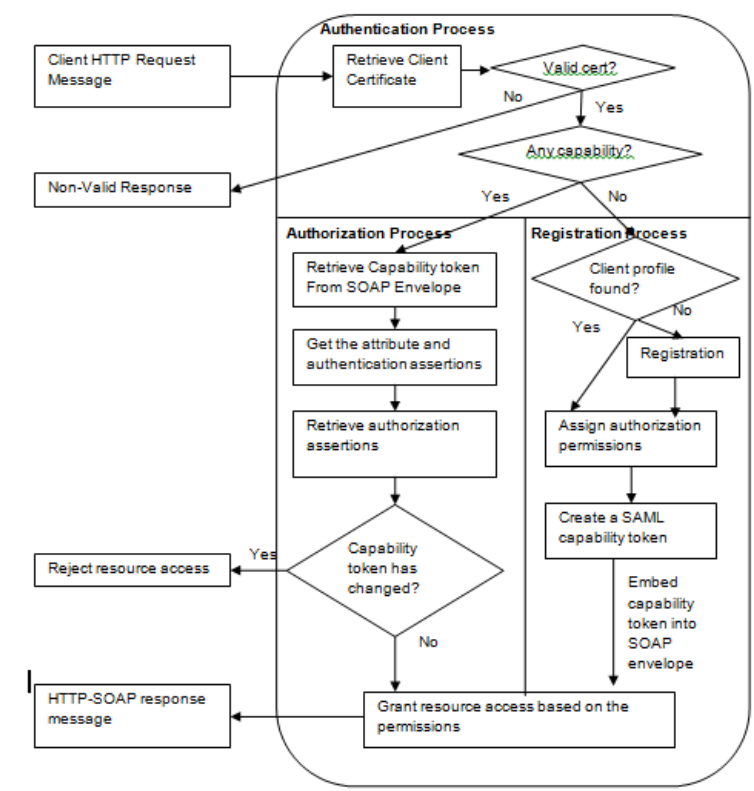

Fig. 2 Access process at resource provider end-point 


\section{EXTENSION TO DRPM}

In this section, we propose an integration of community based trust services to the DRPM. Further, a detailed workflow implementation to provide a fine grained resource protection in DRPM is showed.

\section{A. Community Based Trust Services}

The existing DRPM heavily utilizes a central CA as the third party entity that provides digital certificate for both client and resource provider. A certificate is required to certify the authenticity of an entity which is vital before any transaction occurs. In order for a certificate to be accepted, CA must be trusted by both client and resource provider. Although the utilization of CA is essential for the entity verification, several detriments could possibly limit such implementation in a DE environment. First, the implementation of CA creates barriers to entry for the Small and Medium Enterprises (SMEs) due to the cost ineffectiveness and hectic audit process.

In its practical implementation, an enterprise is required to pay a yearly subscription fee to the CA provider for each certificate that is issued for single enterprise service. Further, a complex audit process is conducted annually to verify the identity of an enterprise. Due to these reasons, the CA certificate is mainly utilized by a small number of large enterprises while hindering such implementation for the SMEs. Note that, this situation further deviate the core purpose of a DE concept which is to enable a greater involvement of SMEs by allowing SMEs to actively engage, collaborate, and gain advantage from an open socio economic environment $[16,17]$.

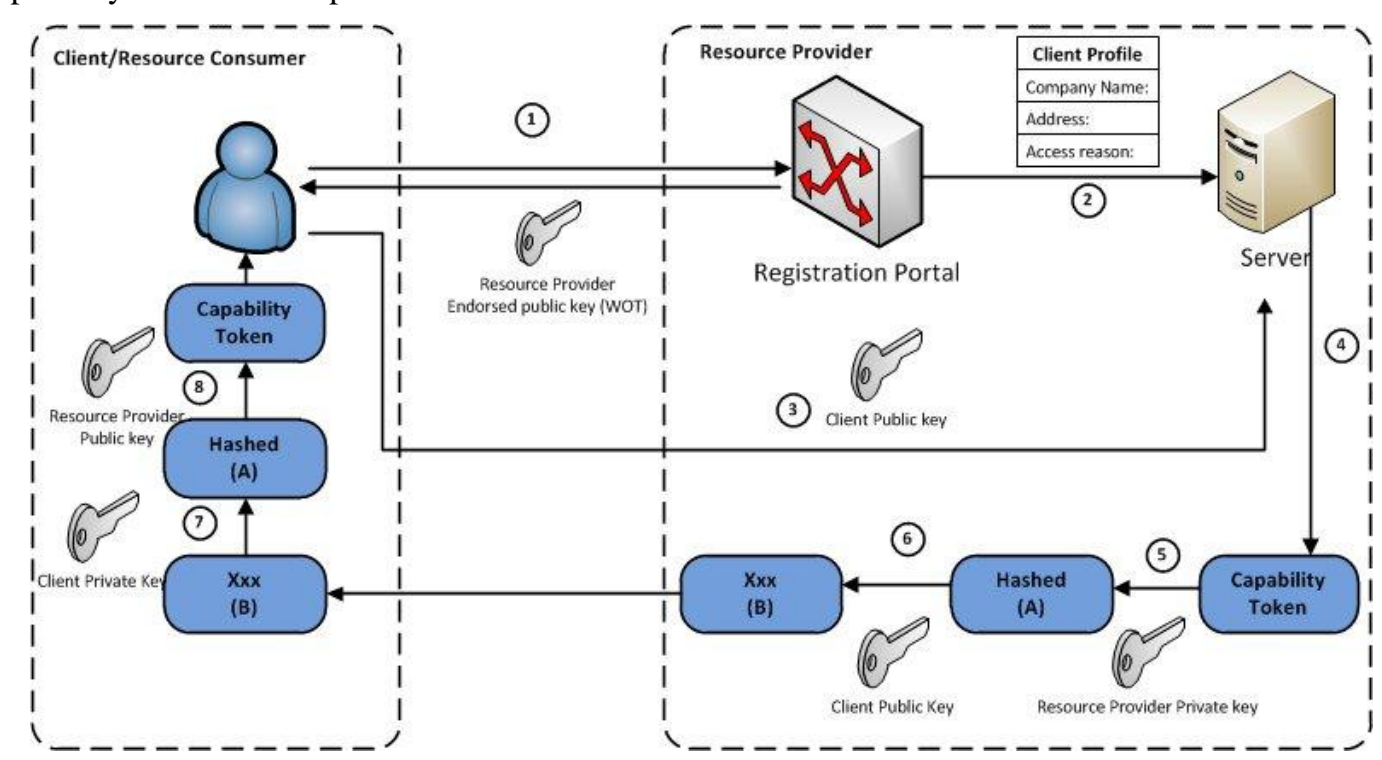

Fig. 3 DRPM secured registration workflow

Second, there is a significant risk of single point failure and CA subversion. The availability of the entire DE environment is threatened when a failure occurs on CA. This failure will yield a substantial impact on situations such as when a joining entity requires a certificate or when certificate is expired. On another case of CA subversion, resource protection may be compromised if a hacker is able to obtain an authentic entity certificate from the CA. For instance, this was the case in [18]. To address the above concerns, the idea presented in this paper is to integrate the community trust services, such as Web of Trust (WoT) [19] to DRPM workflow, particularly in the client registration process. We present this idea as an alternative approach for protecting resources in a DE environment.

Web of Trust (WoT) is a community endorsed certificate which provides a decentralized trust management in a digital community. In WoT, there is no central authority (such as CA) that every entity trust, instead each entity is able to sign others certificates or public keys to build an interconnected web of public keys. The identification of an entity is provided primarily by his public key which is digitally signed by any number of "introducers". Three degree of trustworthiness is introduced to reveal the reliability of the entity public key certificate: undefined, marginal and complete. Final decision for trusting the entity is rely on the user after examining the degree of trustworthiness. The prominent application of WoT is in Pretty Good Privacy (PGP) [20], which is used extensively to secure emails.

\section{B. Securing Registration Workflow}

The DRPM registration portal is used to generate a client profile during the initial resource provisioning. This registration portal also captures the client information and possibly the reasons for accessing the resources. The registration process comprises of three main stages: client registration, public key exchanges, and secure transfer of capability token. WoT is utilized to identify the authentic resource provider based on the community endorsed public key certificate. The Public Key Infrastructure (PKI) is used to provide a secure communication between the client and resource provider. Figure 3 shows the principal workflow for securing three stages of registration process. The registration steps are detailed below: 
1. A new client contacts the resource provider for requesting a resource. Resource provider sends its WoT endorsed public key to the client. Once the client determines and accepts the trustworthiness of the public key, he stores the resource provider trusted public keys and fills his information on the registration portal.

2. After the client information is filled, the registration portal will build a unique client profile which identify the client, and send this client profile to the repository server.

3. Resource provider then requests for client certificate and stores the client public key on its repository. If required, WoT verification could be performed on client certificate to ensure the trustworthiness of the client.

4. Similar to the registration process explained in figure 3, resource provider generates a client capability token based on client's allowed permissions.

5. Resource provider uses its own private key to sign the capability token. SHA Algorithm [21] is also used to hash the capability token. This process enhances the integrity of capability token over the untrusted network.

6. Resource provider then uses client's public key, received from step 3, to encrypt the hashed message and send it to client end-point.

7. Client uses his own private key to decrypt the encrypted capability token. This process further ensures the confidentiality of capability token. A capability token has been breached if client cannot decrypt the message.

8. Client then uses resource provider public key to generate the capability token from the hashed message. This process further ensures that the client receives the capability token from the genuine resource provider unchanged.

Note that at the final step of registration process, the client will have the retrieved capability token and public key from the resource provider. The capability token and resource provider public key are stored in client repository for future communication.

\section{Fine-grained Resource Access Workflow}

Once a client has been successfully registered with the resource provider, client will present his capability token to the resource provider on every access request. The capability token which contains client assertions and authorization permissions is primarily used as a base by the resource provider for granting the resource access. Resource provider utilizes client's capability token to authenticate and authorize client access. Three foremost protection requirements for the resource access are the identification of resource provider, secured transfer of capability token, and authentication of a requesting client. A detailed workflow that ensures security protection on each resource access is provided in figure 4 .

The steps are as follow:

1. Client looks at his repository for his intended resource provider capability token. He then retrieves this capability token from client repository. The capability token contains the client access permissions and the resource URL. At this stage, the client also determines a symmetric pass key which will be shared with the resource provider.

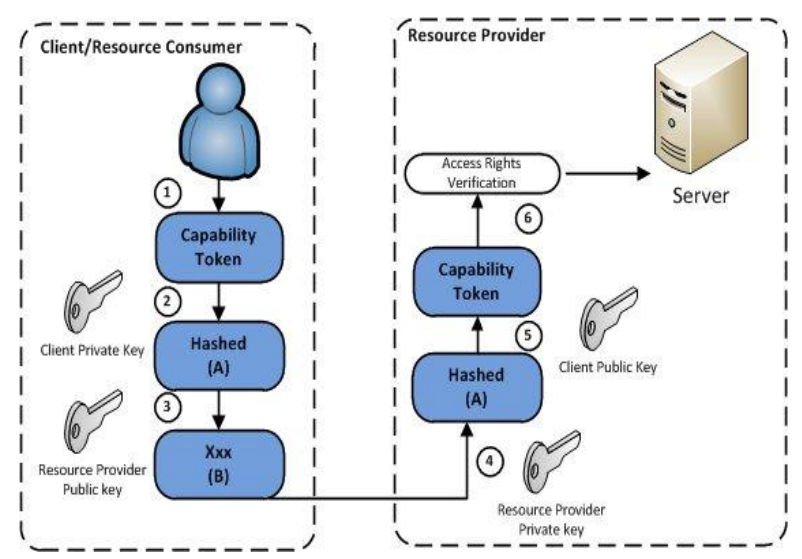

Fig. 4 DRPM resource access protection

2. Client uses his private key to sign the capability token. The signing process is essential to uphold the nonrepudiation of capability token.

3. Client then encrypts the signed capability token using resource provider public key and he sends the encrypted message to the resource provider.

4. When resource provider received the encrypted message, it uses its own private key to de-crypt the message and retrieve hashed capability token.

5. Resource provider then verifies the signature of capability token using the client public key. It then verifies the integrity of the capability token by generating the hash number from capability token using the SHA Algorithm.

6. Resource provider retrieves the access permissions listed in capability token. Further, a symmetric pass key is also obtained at this stage which is used in further communication between client and server during the session. . This process is discussed below.

Note that, on the step 1 of the workflow the client determines a symmetric pass key. This pass key will be utilized to generate a symmetric key for further communication after the capability token authentication and authorization processes is valid. In an event where the capability token is stolen due to the man-in-middle attack [22], the unauthorized entity will still not be able to access the resource due to the symmetric key passphrase that is shared between the legitimate client and resource provider only.

PKI is extensively utilized during the DRPM resource workflow. The other party public key retained by both client and resource provider during the registration process is reused to provide the confidentiality and integrity of capability token. PKI is primarily adopted during the initial handshake and capability token transfer. Due to the limitation of PKI which requires higher computation process, we suggest the utilization of symmetric key for transferring the data after the 
authentication and authorization process. The symmetric pass key can be incorporated into the capability token message before encrypting with the client's private key. Resource exchange is then encrypted by this session key over the untrusted network.

\section{DRPM EXTENSION EVALUATION}

The adoption of WoT into a DE environment is an alternative approach to ensure that the confidentiality, integrity and authentication of resources are being sustained when there is a non-existence of central CA. A DE virtual community comprises multiple interacting entities that mutually trust each other. Such community is an appropriate medium for the implementation of WoT. The similarity between DE concept and WoT lies on the power manifestation of the trusted virtual community, on which each entity provides the verification or endorsement to another entity. Further utilization of the WoT community involvement also facilitates the evolution concept of DE. WoT allows new entities or services to be trusted by the community without a procedure to follow the registration process on a central CA, while the outdated entities or services disappears without administrative overhead on a central CA.

As the decentralized trust mechanism in DE environment matures, the occurrences of $\mathrm{CA}$ as a single certificate provider can be minimized. Several research in [23] and [24] notably presents the decentralized trust mechanism in virtual communities. Further, the elimination of CA is also adopted by JXTA overlay [25] for securing the peer-to-peer networking. In DRPM, the decentralized trust mechanism will therefore enhances the verification of genuine resource provider during registration process. In addition to WoT extension, the utilization of PKI further ensures a fine grained access protection on the DRPM registration and resource access workflow. Public keys of both client and resource provider are being exchanged during the registration process. These keys are retained and re-used for the future resource access. In any case of public key is being compromised on which the generation of a new key is required, this key will be distributed to the designated business entities.

\section{CONCLUSION AND FUTURE WORK}

This paper has highlighted the essential requirements for protecting the resources in a DE environment. Several limitations of the central authentication and authorization process to uphold the resource confidentiality and integrity are shown. Further, we have reviewed the concept of our previously proposed Distributed Resource Protection Mechanism (DRPM). We further extend the DRPM to include the community trust services to provide the identification services. Such proposed approach is vital, in particular when there is non-existence of a central CA. The characteristic of Web of Trust (WoT) is similar to the DE environment on which each entity interacts and supports each other to create a digital community. These entities establish mutual trust and further endorse the authenticity of other entities. Further, we detail the utilization of Public Key Infrastructure (PKI) in DRPM workflows to further enhance the security protection of the resources. Two main workflows of DRPM, the registration and resource access workflows are specifically showed on which PKI is utilized to uphold the confidentiality and integrity of the resources. Future work on the implementation of the presented idea will be conducted.

\section{REFERENCES}

[1] DBE. (2003). The Digital Business Ecosystem: an Introduction to the DBE Project. Available: http://www.digitalecosystems.org /cluster/dbe/dbe_summary_cc.pdf.

[2] H. Boley and E. Chang, "Digital Ecosystem: Principles and Semantics," presented at the 2007 Inaugural IEEE International Conference on Digital Ecosystems and Technologies (IEEE DEST 2007), Cairns, Australia, 2007.

[3] E. Chang, et al., "The vision of DEBI Institute: digital ecosystems and business intelligence: Digital Ecosystem and Business Intelligence Institute," DEBII, Perth, Australia2006.

[4] G. Briscoe, et al., "Biology of applied digital ecosystems," presented at the the Inaugural IEEE International Conference on Digital Ecosystems and Technologies (IEEE DEST 2007), Cairns, Australia, 2007.

[5] G. Briscoe and S. Sadedin, "Digital Business Ecosystem," European Commission, Natural Science Paradigms, 2007.

[6] F. Nachira, et al., "Towards a network of digital business ecosystems fostering the local development," Bruxelles, Discussion Paper2002.

[7] J. M. Seigneur, "Demonstration of security through collaborative in digital business ecosystem," in Proceedings of the IEEE SECOVAL Workshop, Athens, Greece, 2005.

[8] J. Novotny, et al., "An online credential repository for the Grid: MyProxy," in Proceedings of the IEEE Tenth International Symposium on High Performance Distributed Computing (HPDC-10), San Fransisco, USA, 2001.

[9] L. Pearlman, et al., "A community authorization service for group collaboration," in Proceedings of the Third International Workshop on Policies for Distributed Systems and Networks (POLICY'02), California, USA, 2002.

[10] M. Thompson, et al., "Certificate-based access control for widely distributed resources," in Proceedings of the 8th conference on USENIX Security Symposium, Washington DC, 1999.

[11] I. Pranata and G. Skinner, "Managing Enterprise Authentication and Authorization Permissions in Digital Ecosystem," presented at the 3rd IEEE International Conference on Digital Ecosystems and Technologies (DEST '09), Istanbul, Dubai, 2009.

[12] I. Pranata and G. Skinner, "Digital Ecosystem Access Control Management," WSEAS Transactions on Information Science and Applications, vol. 6, pp. 926-935, 2009.

[13] S. J. Mullender and A. S. Tanenbaum, "The design of a capability based distributed operating system," The Computer Journal, vol. 29, pp. 289-299, 1984.

[14] W3C. (February 2011). Extensible Markup Language (XML). Available: http://www.w3.org/XML/

[15] J. Weise, "Public Key Infrastructure Overview," Sun Microsystem, Sun BluePrints Online2001.

[16] E. Commission. (2002). Towards a network of digital business ecosystems fostering the local development. Available: http://www.digital-ecosystems.org/ doc/discussionpape r.pdf

[17] P. Dini, et al., "The digital ecosystems research vision: 2010 and beyond," Position Paper, 2005.

[18] Verisign. (2001, January 2011). Advisory from VeriSign, Inc. Available: http://www.verisign.com/support/advisories/authen ticodefraud.html

[19] A. Abdul-Rahman, "The PGP Trust Model," EDI-Forum, 1997.

[20] P. R. Zimmermann, The Official PGP User's Guide. Massachusetts, USA: MIT Press, 1995.

[21] NIST, "Secure Hash Standard (SHS)," in DRAFT FIPS PUB 180-4, ed: National Institute of Standards and Technology (NIST), 2011 
[22] F. Callegati, W. Cerroni, and M. Ramilli, "Man-in-the-Middle Attack to the HTTPS Protocol," IEEE Security \& Privacy, vol. 7, pp. $78-81,2009$.

[23] G. Skinner and M. Miller, "Managing privacy, trust, security, and context relationships using weighted graph representations.," WSEAS Transactions on Information Science and Applications, vol. 3, pp. 283-290, 2006.

[24] A. Abdul-Rahman and S. Hailes, "Supporting Trust in Virtual Communities," in Proceedings of the 33rd Hawaii International Conference on System Sciences, Hawaii, USA, 2000

[25] J. Arnedo-Moreno, K. Matsuo, L. Barolli, and F. Xhafa, "Secure Communication Setup for a P2P based JXTA-Overlay Platform," IEEE Transactions on Industrial Electronics, 2010. 